FACULDADE DE CIÊNCIAS ECONÔMICAS DA UFRGS Análise
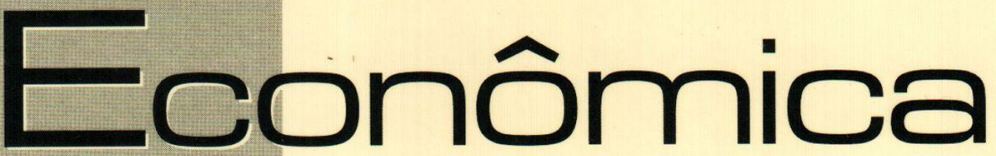

FLUTUACÃO CAMBIAL E METAS INFLACIONÁRIAS EM

ECONOMIAS EMERGENTES

OTAVIANO CANUTO E MárCIO HOLLAND

ATEORIA DE FORMACĆOO DE PRECOS E A TEORIA DOS PREÇOS DE

PRODUCAO

LOIZALBERTO ESTEVES

AECONOMIA EVOLUCIONISTA: UM CAPITULOSISTEMMICO DA

TEORIA ECONOMICA?

HUGOE. A. DA GAMACERQUEIRA

RELAÇ̃O INFLAÇÃO-PRODUTO NO BRASIL NO PERIODO PÓS-

REAL

MARCOSC. HOLANDA

DIVIIDA PÚBLICAMOBILLÍRIA FEDERAL BRASILEIRA: HISTÓRIA RECENTE E PERSPECTIVAS EXPLOSIVAS

ROGÉRIO MEURER E ROBERT WAYNE SAMOHYI

A CEPAL EA INTEGRACÃO REGIONAL LATINO-AMERICANA JACQUELINE A. HERNANDEZ HAFFNER

POPPER, HAYEK EA (IM)POSSIBILIDADE DE PREDIÇŌES ESPECIFICAS EM CIEENCIAS SOCIAIS

BRENA PAULA MAGNO FERNANDEZ

DINÂMICA RECENTE DO PROCESSO DE INCUBACG̃O DE

EMPRESAS DE BASE TECNOLOGICA NO BRASIL

EDUARDO GONÇALVES

TRIBUTACĀO COM SACRIF́CIO EQUUITATIVO: O CASO DO IMPOSTO DE RENDA PESSOA FISICA

STEFANO FLORISSI E EDUARDO PONTUAL RIBEIRO

A NOVATEORIA DO IMPÉRIOE AS VELHAS TEORIAS DO

IMPERIALISMO

GENTIL CORAZZA

Ô ABREALAS - A NOVA INSERÇÃO DO BRASIL NA ECONOMIA MUNDIAL

EMLLLANO LUIS KLEN

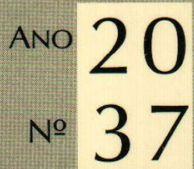

Março, 2002 
UNIVERSIDADE FEDERAL DO RIO GRANDE DO SUL

Reitora: Profa. Wrana Maria Panizzi

FACULDADE DE CIÊNCIAS ECONÔMICAS

Diretor: Prof. Pedro Cézar Dutra Fonseca

CENTRO DE. ESTUDOS E PESQUISAS ECONÔMICAS

Diretor: Prof. Gentil Corazza

DEPARTAMENTO DE CIÊNCIAS ECONÔMICAS

Chefe: Prof. Luiz Alberto Oliveira Ribeiro de Miranda

DAPARTAMENTO DE CIÊNCIAS CONTÁBEIS E ATUARIAIS

Chefe: João Marcos Leão da Rocha

CURSO DE PÓS-GRADUAÇÃO EM ECONOMIA

Coordenador: Prof. Eduardo Pontual Ribeiro

CURSO DE PÓS-GRADUAÇÃO EM ECONOMIA RURAL

Coordenador: Prof Jalcione Almeida

CONSELHO EDITORIAL: Carlos G. A. Mielitz Netto (UFRGS), Eduardo A. Maldonado Filho (UFRGS), Eduardo P. Ribeiro (UFRGS), Eleutério F. S. Prado (USP), Eugênio Lagemann (UFRGS), Fernando Cardim de Carvalho (UFRJ), Fernando Ferrari Filho (UFRGS), Fernando de Holanda Barbosa (FGV/RJ), Flávio Vasconcellos Comim (UFRGS), Gentil Corazza (UFRGS), Giácomo Balbinotto Neto (UFRGS), Gustavo Franco (PUC/RJ), Jan A. Kregel (Università di Bologna e John Hopkins University), João Rogério Sanson (UFSC), Joaquim Pinto de Andrade (UnB), Jorge Paulo Araújo (UFRGS), Juan H. Moldau (USP), Marcelo S. Portugal (UFRGS), Maria Alice L.ahorgue (UFRGS), Paul Davidson (University of Tennessee), Paulo Dabdab Waquil (UFRGS), Pedro Cézar Dutra Fonseca (UFRGS), Philip Arestis (South Bank University), Roberto C. Moraes (UFRGS), Ronald Otto Hillbrecht (UFRGS), Sabino da Silva Porto Jr. (UFRGS), Stefano Florissi (UFRGS), Werner Baer (Univ, of Illinois at Urbana-Champaign).

COMISSÃO EDITORIAL: Eduardo Augusto Maldonado Filho, Fernando Ferrari Filho, Gentil Corazza, Marcelo Savino Portugal, Paulo Dabdab Waquil; Roberto Camps Moraes.

EDITOR: Fernando Ferrari Filho

EDITOR ADJUNTO: Gentil Corrazza

SECRETÁRIA: Vanessa Hoffmann de Quadros

REVISÃO DE TEXTOS: Vanete Ricacheski

FUNDADOR: Prof. Antônio Carlos Santos Rosa

Os materiais publicados na revista Análise Econômica são da exclusiva responsabilidade dos autores. É permitida a reprodução total ou parcial dos trabalhos, desde que seja citada a fonte. Aceita-se permuta com revistas congêneres. Aceitam-se, também, livros para divulgação, elaboração de resenhas e recensões. Toda correspondência, material para publicação (vide normas na terceira capa), assinaturas e permutas devem ser dirigidos ao seguinte destinatário:

PROF FERNANDO FERRARI FILHO

Revista Análise Econômica - Av. João Pessoa, 52 CEP 90040-000 PORTO ALEGRE - RS, BRASIL Telefones: (051) 316-3348 e 316-3440 - Fax: (051) 316-3990

E-mail: rae@vortex ufrgs br

Análise Econômica

Ano 20, n 37 , março, 2002 - Porto Alegre

Faculdade de Ciências Econômicas, UFRGS, 2000

Periodicidade semestral, março e setembro.

1. Teoria Econômica - Desenvolvimento Regional Economia Agrícola - Pesquisa Teórica e Aplicada -

Periódicos. I. Brasil.

Faculdade de Ciências Econômicas,

Universidade Federal do Rio Grande do Sul.

CDD 330.05

CDU 33 (81) (05) 


\title{
Flutuação cambial e metas inflacionárias em economias emergentes
}

Otaviano Canuto ${ }^{*}$ e Márcio Holland ${ }^{*}$

\begin{abstract}
Resumo: Este trabalho pretende apresentar um modelo-síntese para compreensão de flutuações cambiais sob um regime de metas de inflação, a partir de um modelo Mundell-Flemmig expandido. Procuramos destacar as relações entre a escolha de uma meta inflacionária com o comportamento da taxa de juros e do produto, conforme esperado por uma política monetária ótima. Nossa conclusão é a de que a estratégia de metas inflacionárias está fortemente associada à escolha de regimes de câmbio mais flexíveis, e que deve se constituir em sucesso em estabilizar o comportamento de preços em níveis relativamente baixos, o que implicaria alguma trajetória de crescimento do produto, na ausência de choque de oferta, mas não necessariamente de taxa de juros baixas e de taxa de câmbio relativamente pouco volátil.
\end{abstract}

Palavras-Chave: Modelo Mundell-Fleming, Metas de Inflação, Taxa de Câmbio flutuante.

\begin{abstract}
This article presents a synthetic model to understand the exchange rate fluctuation under inflation targeting regime, according modified Mundell-Fleming model. We highlighted the relationship between the choice of target to the inflation and the behaviour of the interest rate, as expected in optimum monetary policy. We can conclude that the inflation targeting strategy is strongly linked to floating exchange rate regimes and that it reaches success only in stabilizing price in low level, what implicate in growth of the real product, if supply shocks does not take place, but it does not necessarily implicate in a macroeconomic context of low interest rate and exchange rate little volatile.
\end{abstract}

Key words: Mundell-Fleming Model, Inflation Target, Floating exchange rate.

\footnotetext{
* Professor da USP e Pesquisador CNPq.E-mail: ocanuto@uol.com.br.

“" Professor do IE/UFU e Pesquisador CNPq. E-mail: mholland@ufu.br. Ambos os autores agradecem o apoio financeiro do CNPQ
} 
Modelos de comportamento da taxa de câmbio são comumente associados ao Modelo Mundell-Fleming, seja com preços flexíveis, seja na versāo de Dornbusch (1976) para preços rígidos, com hipótese sobre mobilidade de capital imperfeita, mas sem prêmio de risco, e com o teorema de paridade de poder de compra válido para o longo prazo, qualquer que seja o desvio de curto prazo na trajetória da taxa de câmbio. Com a adoção de regimes de câmbio mais fixo em um conjunto significativo de economias emergentes, os desenvolvimentos teóricos na direção deste modelo foram substituídos por modelos de banda de flutuação estáveis ou móveis, de crises cambiais, de segunda e terceira geração, com incorporação de noçōes sobre expectativas auto-realizáveis e com incorporação de alguma noção sobre prêmio de risco.

As recentes crises cambiais impuseram uma mudança de rota nas estratégias de políticas monetárias em muitas economias emergentes. Dado o sucesso relativo das estratégias de metas de inflação, a implementação de regimes de câmbio com flutuação independente ou administrada passa a se associar com mecanismos típicos de metas inflacionárias. Nosso objetivo com este trabalho é o de apresentar um modelo-síntese de interpretação de flutuação cambial sob regime de metas de inflação. Apresentaremos, para tanto, na próxima seção um conjunto de elementos para reflexão sempre que se discute inflation targeting. Em seguida, recuperaremos os mecanismos de ajustamento macroeconômicos em economia aberta, sob condiçōes de mobilidade de capital, a partir de um Modelo Mundell-Fleming com inflação.

Na última seção, apresentaremos um sistema de equações que expressam a dinâmica da inflação sob condições de expectativas adaptativas ou racionais, a partir da Curva de Phillips e de situações possível para a execução de políticas antiinflacionárias ótimas, bem como alguma regra de comportamento da taxa de câmbio, associada ao comportamento da taxa de juros, tal que mecanismos de transferências inflacionárias através da taxa de câmbio se estabeleçam em padrões adequados à meta inflacionária, guardados os mecanismos de ajustamento em situação de desvios da meta de inflação. Demonstra-se que, se por um lado, a estratégia monetária de metas de inflação é bem-sucedida em sustentar a taxa de inflaçāo baixa, ou mesmo garantir algum crescimento nas taxas de produto, o mesmo sucesso não pode ser obtido para baixar as taxas de juros e evitar perturbações no mercado cambial. 


\section{Alguns fatos estilizados}

Nitidamente, a implementação de regimes de metas inflacionárias (Inflation Targeting) tem como um dos objetivos centrais a eliminação do viés inflacionário presente em regimes de câmbio mais flexíveis. De fato, metas inflacionárias não são compatíveis com regimes de câmbio fixo, dado o caráter endógeno das políticas monetárias sob regime de câmbio fixo. Ou ainda, "Difficulties in conducting monetary policy using an exchange rate peg or some monetary aggregate as the main intermediate target led a number of industrial countries in the 1990s to adopt a framework for monetary policy that has become know as inflation targeting" (Masson, Savastano E Sharma, 1997, p. 3). Assim, tal estratégia deve se constituir em um importante instrumento a auxiliar na estabilidade de preços, seja na obtenção de baixa inflação, seja na menor expectativa inflacionária. Novamente, a discussão sobre regras versus discrição na execução da política monetária volta-se para um problema de graus de discrição. Há, aqui, que considerar um aspecto importante deste problema: o problema da credibilidade na politica (conhecido também como "problema de inconsistência temporal" - time inconsistency problem), conforme apontado por Kydland $\mathcal{Z}$ Prescott (1977), Barro $\mathcal{Z}$ Gordon (1983), entre outros. Afinal, as metas inflacionárias podem ser vistas muito mais como uma estratégia com considerável grau de discrição do que como uma regra de política econômica.

Se a inflação se manteve realmente muito baixa em economias industriais que adotaram o regime de metas inflacionárias, também em economias que não o implementaram a inflação se mantém baixa (Debelle,1997 e Sicsú, 2000). Se o uso de metas para a inflação representa um passo à frente na evolução da estratégia de política monetária, também é fato que tais metas propiciam um elemento adicional devido a problemas de falta de credibilidade (lack of credibility), característicos, por exemplo, de economias que sofrem da transição forçada de regimes de câmbio mais pegged para regimes mais float. "Consequently, one of the main reasons for adopting inflation targets is to enhance the credibility of monetary policy. In one sense, inflation targets are track on which these countries seek to build their record of low inflation and monetary policy credibility. (Debelle, 1997, p. 4).

Uma outra razão muito comumente apontada para a adoção de um regime de metas de inflação é o próprio viés inflacionário potencial advindo da execução de políticas monetárias (Fisher,1993 $\mathcal{B}$ Barro, 1995). Neste caso, metas inflacionárias removem o vício das Autorida- 
des Monetárias em adotar políticas monetárias discricionárias (Masson, Savastano 3 Sharma, 1997). Afinal, o Banco Central faz uma previsão de trajetória futura da inflação, tal que a diferença entre a taxa prevista e a taxa observada de inflação exigiria ajustamento nos instrumentos de política monetária. Assim, o papel das metas de inflação é prover uma âncora nominal, tanto sobre a inflação presente quanto sobre expectativas de inflação, com transparência das políticas econômicas e com mecanismos de prestação de contas (accountability) e de ajustamento em caso de desvio da meta. Sob estas condições, dada a defasagem nos efeitos da política monetária, um regime de metas inflacionárias deve, por princípio, constituir um modelo de inflação do tipo forward-looking.

Sobre os efetivos das metas inflacionários, a conclusão de Debelle (1997, p. 28), é muito pouco conclusiva.

To move to an inflation targeting framework in a number of countries appears to been sucessfull in reducing inflation. Inflation has been reduced to low levels and has remained there through the expansionary phase of cycle. It is difficult to assign the credit for this solely to the adoption of inflation targeting framework, given that inflation also fell in number of other countries that did not explicitly practice inflation targeting.

Em Netto (1999, p. 373), “Talvez seja um pouco prematuro saudar o enorme sucesso da inflation targeting [...] De qualquer forma, representa um grande avanço na formulação da política monetária". Mais ainda, este regime "está longe de se concentrar exclusivamente sobre a taxa de inflação, porque tem de considerar, também, os seus efeitos sobre a flutuação do PIB, o que certamente é uma vantagem" (Netto, 1999, p. 269).

Debelle (1997, p. 9) levanta um conjunto de metas e políticas macroeconômicas que poderiam, sob algumas condições e hipóteses, serem fortemente inconsistentes com as metas inflacionárias. Entre elas, metas de pleno emprego não necessariamente seriam inconsistentes com metas de inflação. "In long run, the achievement of the inflation target may be the best contribution that monetary policy can make to the full employment objective. In the short run, however, there may be a tradeoff between the two objectives". Não se coloca apenas a questão da inconsistência de metas, como também problemas associadas com o fato de que metas de inflação produzem instabilidade do produto. Neste caso, Jadresic (1999, p. 22) conclui que "inflation targeting and output stabilization may be made compatible by targeting na inflation indicator 
that relieves monetary policy from the burden of trying to offset the of antecipated price shocks on headline inflation".

Há, assim, que se discutir se a inflação se mantém baixa, em níveis desejáveis pelos objetivos macroeconômicos assumidos pelas Autoridades Monetárias, somente com o recurso de um regime de metas in. flacionárias, ou se, guardada a generalidade, as metas inflacionárias só seriam eficazes quando os componentes típicos de um processo de inflação crônica já se dissiparam, como é o caso de mecanismos de indexações tipicamente inerciais, ou de indexações conduzidas com expectativas tipo forward-looking. Ao mesmo tempo, as metas inflacionárias só seriam críveis em dois sentidos particulares. Primeiro, porque qualquer meta macroeconômica deveria ser claramente subordinada à meta de inflação. Este é o caso de metas de produto, que devem ser consistentes com inflações não aceleracionistas (Greene, 1996, p. 780). Segundo, há o fato de que o regime de metas inflacionárias levaria a políticas discricionárias tais que as Autoridades Monetárias poderiam expandir o produto, no curto prazo, através de políticas expansionistas. "Put another way, potencial inconsistencies between the inflation target and the preference to raise output beyond potencial may render inflation targeting neither credible nor enforceable"(Greene, 1996 p. 781). Deste modo, "discretionary policies result in a higher average level of inflation but do not improve output" (Greene, 1996, p. 787).

Há, certamente, muitas defesas ao regime de metas de inflação, todas centradas na relativa autonomia adquirida na execução de política monetária e, por conseguinte, na superioridade desta estratégia frente outras tidas como rígidas, sob regimes de câmbio fixo. Oposta à estratégia da dolarização plena (ausência completa de espaço para política monetária somada ao fim do Banco Central como emprestador de última instância), ou do comitê de moeda (em que o Banco Central se transforma numa caixa de conversão), a estratégia de metas de inflação, também é defendida por aqueles que pregam a transparência da gestão monetária aliada à simplicidade de compreensão do público. Contudo, há muitas críticas a esta estratégia de política monetária. Conforme Mishkin 8 Savastano (2000 p. 34),

Critics of inflation targeting have noted at least seven disadvantages of this monetary policy strategy. Four of those disadvantages - that inflation targeting is too rigid, that it is tantamount to full discretion, that it necessarily increases output instability, and that it hurts economics growth - we believe are misplaced. The fifth disadvantage, that inflation targeting can only produce weak central bank accountability because is hard to control and because there are long lags from the monetary instruments to the inflation outcome, is a serious one indeed. 
Muitas economias vêm adotando, algumas há dez anos ou mais, uma estratégia de política monetária que combina regimes de câmbio mais flexíveis com metas inflacionárias, ou com metas monetárias explícitas. Note-se, ainda, com ajuda do anexo 1, a forte preferência atual por regimes de câmbio com flutuação independente (em torno de 51 países), mas, em sua maioria com algum objetivo estratégico (seja agregado monetário, seja inflação), e em sua grande maioria, mas fracamente associado à estratégia de objetivos macroeconômicos, com algum programa econômico respaldado pelo FMI. Este o caso de um único país, o Brasil, que, ao adotar um regime de câmbio mais flexível, com objetivos explícitos para inflação (as metas de inflação), apresenta um programa econômico sob monitoramento do FMI. Todos os demais países que apresentam algum programa com o FMI estão sob regime de câmbio com flutuação mais independente, mas não sob metas monetárias ou inflacionárias, e são, em sua grande maioria, economias pequenas (como Albânia, Armênia, Filipinas, Madagascar etc.) ou são, ainda, economias que viveram, recentemente, alguma crise cambial (como o México, a Tailândia, a Indonésia, a Rússia, e o próprio Brasil, entre outras).

Voltemo-nos à estrutura típica de um modelo de metas de inflação, conforme amplamente divulgado na literatura. Estes modelos, em geral, são compostos pelos seguintes ingredientes: 1) uma noção clara sobre os mecanismos de ajustamento macroeconômico em economia aberta, sob condições de mobilidade de capital; 2) um sistema de equações que expressem a dinâmica da inflação sob condições de expectativas adaptativas ou racionais, a partir da Curva de Phillips e de situações possível para a execução de políticas antiinflacionárias ótimas; 3) alguma regra de comportamento da taxa de câmbio, associado ao comportamento da taxa de juros, tal que mecanismos de transferências inflacionárias através da taxa de câmbio se estabeleçam em padrões adequados à meta inflacionária; e 4) mecanismos de ajustamento em situação de desvios da meta de inflação. 


\subsection{O modelo básico}

A economia aqui modelada está composta por cinco mercados, tratados de forma agregada: o mercado de bens e serviços; o mercado monetário; o mercado de títulos financeiros; o mercado de trabalho e o mercado cambial. Ao incorporar este último - considerando a conta de capitais do balanço de pagamentos e a mobilidade internacional de capitais - o modelo Mundell-Fleming abre ao exterior a macroeconomia estilizada nos modelos IS-LM com preços flexiveis, ou seja, a versão de macroeconomia com oferta e demanda agregadas que serve de referência nos manuais introdutórios de macroeconomia fechada.

O modelo descrito neste item contém nove equações ou identidades, representando as posições de equilíbrio nos mercados. Por seu turno, 11 variáveis endógenas são apontadas, o que abre a necessidade de mais duas relações para tornar possível a determinação de pelo menos uma posição de equilíbrio geral dos mercados. As âncoras nominais impostas em seguida - sobre a taxa de câmbio ou o volume real de meios de pagamento - fecham o modelo sob formas distintas.

Cumpre também lembrar que, na hipótese de quatro dos cinco mercados estarem em equilíbrio, o $5^{\circ}$ também estará. Dada a restrição orçamentária que vigora nas decisões dos agentes, a presença de igualdade entre compras e vendas em quatro mercados implica necessariamente que os agentes participantes do $5^{\circ}$ mercado também estarão exercendo compras e vendas em iguais magnitudes (a "Lei de Walras"). Qualquer análise de equilíbrio simultâneo em mercados deve considerar como redundante algum entre eles. Em nosso caso, tomaremos como redundante a condição de equilíbrio no mercado de títulos: os componentes de oferta e demanda de títulos estarão presentes nos demais mercados, mas o equilíbrio simultâneo destes torna redundantes, para a resolução do modelo, as informações contidas no equilíbrio daquele mercado.

\footnotetext{
1 O modelo Mundell-Fleming foi construído a partir de trabalhos de Robert Mundell - prêmio Nobel de economia em 1999 - e de Marcus Fleming, nos anos 60, estendendo o tradicional modelo IS-LM para o contexto de economias abertas. Para sua comparação com a análise keynesiana de economias abertas - cronologicamente anterior ao Mundell-Fleming - bem como com as abordagens que se seguiram, ver Gonçalves et al. (1998). Aqui tomaremos como referência suas versões que incluem a flexibilidade de preços e salários. Para o Mundell-Fleming completo, veja McCallum (1996).
} 
Numa economia aberta, o equilibrio no mercado de bens e serviços corresponde à convergência entre a geração de renda no processo produtivo e a demanda agregada pelo correspondente produto. Tipicamente supõe-se algo como o contido na equação 1 , na qual $Y$ vale para renda real agregada do país, $r$ representa a taxa real de juros que influencia os gastos reais com consumo (C) e investimento (I), $\overline{\mathrm{G}}$ é o montante real de gastos governamentais considerados exógenos $\mathrm{e} \mathrm{X}$ corresponde ao saldo comercial.

Este saldo comercial cresce com a renda real no resto-do-mundo $\left(\bar{Y}^{*}\right)$, exógena ao modelo, a qual influencia de modo positivo as exportações, enquanto a renda real doméstica faz crescerem as importações. Por seu turno, quando a taxa real de câmbio (S) definida na equação 2 se eleva (cai), desvalorizando-se (valorizando-se) a moeda local em termos reais, o saldo comercial aumenta (diminui). Supor positivo este sinal de $\partial \mathrm{X} / \partial \mathrm{S}$ equivale a supor a vigência da condição Marshall-Lerner, ou seja, em última instância, após uma desvalorização cambial, o efeito de diminuição do saldo decorrente da expansão doméstica da demanda agregada e da deterioração nos termos de troca é mais que compensado por aumentos na quantidade exportada (Gonçalves et al., 1998, p. 214-18).

(1) $\mathrm{Y}=\mathrm{C}(\mathrm{Y}, \mathrm{r})+\mathrm{I}(\mathrm{Y}, \mathrm{r})+\overline{\mathrm{G}}+\mathrm{X}\left(\mathrm{S}, \overline{\mathrm{Y}}^{*}, \mathrm{Y}\right)$

com as derivadas $\partial \mathrm{Y} / \partial \mathrm{C}, \partial \mathrm{Y} / \partial \mathrm{I}, \partial \mathrm{X} / \partial \mathrm{S}$ e $\partial \mathrm{X} / \partial \mathrm{Y}^{*}$ todas positivas e $\partial \mathrm{C} / \partial \mathrm{r}$, $\partial \mathrm{I} / \partial \mathrm{r}$ e $\partial \mathrm{X} / \partial \mathrm{Y}$ negativas.

Sendo E, $\mathrm{P}^{*}$ e $\mathrm{P}$, respectivamente, a taxa nominal de câmbio (número de unidades monetárias locais para cada unidade de moeda externa), o nível (exógeno) de preços de produtos no exterior e o nível doméstico de preços, a taxa real de câmbio equivale a:

(2) $\mathrm{S}=\left(\mathrm{E} \cdot \overline{\mathrm{P}}^{*}\right) / \mathrm{P}$

A resolução da equação (1), em termos de Y como a variável determinada pelas demais, provê o equilíbrio no mercado de bens e serviços:

(1a) $Y=A\left(r, S, \bar{Y}^{*}, \bar{G}\right)$

Trata-se da contrapartida da relação IS em uma economia aberta. Supondo-se a vigência da condição Marshall-Lerner, tem-se $\partial Y / \partial \mathrm{r}<0$ e $\partial \mathrm{Y} / \partial \mathrm{S}>0$. Aumentos ou quedas exógenas em $\overline{\mathrm{Y}}^{*}$ e $\overline{\mathrm{G}}$ afetam a renda doméstica na mesma direção em que variam.

Para vários propósitos, vamos supor que o equilibrio relatado em (1a) pode ser aproximado - pelo menos em sua vizinhança -- como uma equação linear em termos dos logaritmos das variáveis. Em (1b), tem-se $y=\ln Y ; s=\ln S$ e $\bar{y}^{*}=\ln Y^{*}$, enquanto $r$ continua representan- 
do a taxa real de juros. Os coeficientes $a_{2}$ e $a_{3}$ são as elasticidades da renda em relação à taxa real de câmbio e à renda real do resto-domundo, ao passo que $\bar{a}$ é o logaritmo natural da soma dos componentes autônomos em relação à renda e juros presentes em $\mathrm{C}, \mathrm{I}, \mathrm{G}$ e X:

(1b) $y=\bar{a}-a_{1} \cdot r+a_{2} \cdot s+a_{3} \cdot \bar{y}^{*}$

Por seu turno, a taxa real de câmbio também pode ser representada como em (2a), com e $=\ln E, \bar{p}^{*}=\ln \bar{P}^{*}$ e $p=\ln P$. Note-se também que, se a condição de equilíbrio (1b) se mantém ao longo do tempo, com y e r constantes, prevalece a condição (3) - na qual $\mathrm{d}(.) / \mathrm{dt}$ representa a derivada da variável no tempo ${ }^{2}$ :

(2a) $\mathrm{s}=\mathrm{e}+\overrightarrow{\mathrm{p}}^{*}-\mathrm{p}$

(3) $(\mathrm{ds} / \mathrm{dt})=(\mathrm{de} / \mathrm{dt})+\left(\mathrm{d} \overline{\mathrm{p}}^{*} / \mathrm{dt}\right)-(\mathrm{dp} / \mathrm{dt})=0$

$\mathrm{Na}$ relação entre o mercado de bens e serviços e o mercado de trabalho, vamos supor que a produtividade média do trabalho (L) cresce, intertemporalmente $(\mathrm{t})$ de modo exógeno, à taxa g, conforme (4). No modelo, o subscrito $(0)$ representará sempre os valores de variáveis no momento inicial de equilibrio.

(4) $\overline{\mathrm{L}}_{0}=\mathrm{L}_{\mathrm{t}} \cdot e^{-\overrightarrow{\mathrm{g} \cdot \mathrm{t}}}$

Implicitamente, os níveis de investimento no mercado de bens e serviços atuam sobre a capacidade instalada, ao longo do tempo, além de seu papel na formação da demanda agregada. O progresso técnico está aqui determinado exogenamente.

Para isolar os efeitos das flutuações no emprego sobre a inflação, objeto da análise, suponhamos exógena a determinação de $\mathrm{N}$, o nível de emprego $(\overline{\mathrm{n}}=\ln \overline{\mathrm{N}})$. A equação 4 a aponta o nível de $Y$ compatível com o equilíbrio no mercado de trabalho, $\mathrm{com}^{3}$

$$
\begin{aligned}
& \overline{\mathrm{l}_{0}}=\ln \overline{\mathrm{L}}_{0} \text { e } \mathrm{l}_{\mathrm{t}}=\overline{\mathrm{l}_{0}}+\overline{\mathrm{g}} \cdot \mathrm{t} \\
& \text { (4a) } \mathrm{y}=\overline{\mathrm{n}}+\overline{\mathrm{l}_{0}}
\end{aligned}
$$

\footnotetext{
${ }^{2} \mathrm{~A}$ arbitragem entre os preços de produtos locais e externos não garante no modelo a Paridade absoluta do Poder de Compra ( $\mathrm{p}=\mathrm{e}+\overline{\mathrm{p}}^{\star}, \mathrm{s}=0$ e $\mathrm{S}=1$ ), mas tão-somente a Paridade em sua forma relativa, se prevalece o equilibrio ao longo do tempo. Sobre estes fundamentos da taxa de câmbio, ver Gonçalves et al. (1998, p. 207-12).

${ }^{3}$ Observe-se que a relação (4a) é compativel tanto com funçōes de produção do tipo Leontieff (coeficientes fixos), quanto com funçōes "bem comportadas", de coeficientes técnicos variáveis. Basta que a relação capital-trabalho se mantenha constante ao longo do tempo, bem como, no segundo caso, que o progresso técnico expresso em (4) seja "neutro" no sentido de Hicks.
} 
A formação de preços de bens e serviços, pelas empresas, corresponde a aplicar uma margem fixa $(\mathrm{T}>1$ ) sobre os custos salariais em termos nominais (W), de modo a cobrir estes e os demais custos, além da obtenção do lucro. As variáveis $\omega$ e $\bar{\tau}$ são os correspondentes logaritmos:

(5) $P=W \cdot T$

(5a) $\mathrm{p}=\omega+\bar{\tau}$

Voltemo-nos agora para os mercados monetário, cambial e de títulos financeiros. Antes de tudo, a identidade (6) define a relação entre a taxa de juros real (r) e a taxa nominal de juros (i), com a diferença correspondendo à taxa de inflação $(\pi \equiv \mathrm{dp} / \mathrm{dt})$ :

(6) $\mathrm{i} \equiv \mathrm{r}+\pi$

O equilíbrio no mercado monetário supõe igualdade entre a disponibilidade local de meios de pagamento, em termos reais, e sua demanda doméstica. Pode-se imaginar uma relação positiva entre a renda da economia e essa demanda, face às necessidades transacionais, bem como uma relação negativa entre tal demanda por saldos monetários reais e a taxa nominal de juros, já que esta significa o custo de oportunidade em termos de ganhos de juros com a retenção de riqueza financeira sob a forma monetária. Façamos também uma aproximação log-linear (equação 7a) do equilíbrio no mercado monetário (equação 7), com M correspondendo à oferta nominal de moeda e $\mathrm{m}$ a seu logaritmo.

(7) $\mathrm{M} / \mathrm{P}=\mathrm{M}^{\mathrm{d}}(\mathrm{Y}, \mathrm{i}) \quad$ com $\partial \mathrm{M}^{\mathrm{d}} / \partial \mathrm{Y}>0$ e $\partial \mathrm{M}^{\mathrm{d}} / \partial \mathrm{r} \leq 0$

(7a) $m-p=\vec{b}+b_{1} \cdot y-b_{2} . i$

Nos mercados cambial e de títulos financeiros, a baixa mobilidade internacional de capitais impōe a igualdade representada em (8) e (8a), onde $\overline{\mathrm{i}}^{*}$ é a dada taxa nominal de juros no exterior, $\overline{\mathrm{k}}$ é um prêmio de risco tomado como exógeno e $\eta \equiv$ (de/dt), com expectativas racionais prevalecendo no equilibrio steady-state, ao longo do tempo. Em (8a), $\overline{r^{*}}$ é a taxa real de juros externa e $\bar{\pi}^{*}$ corresponde à inflação no resto-do-mundo, ambas exógenas ao país em análise.

Afinal, qualquer diferença entre os dois lados de (8) ou (8a) tende a suscitar deslocamentos intensos de capital, dadas as possibilidades de usufruto de rendimentos via arbitragem entre operações locais e no exterior. Há baixa mobilidade de capital, tal que a presença de um prêmio de risco incorpora a imperfeita substitutibilidade entre ativos domésticos e do resto-do-mundo. 
(8) $\mathrm{i}=\overline{\mathrm{i}^{*}}+\overline{\mathrm{k}}+\eta$

(8a) $\mathrm{r}+\pi=\overline{\mathrm{r}}^{*}+\overline{\mathrm{k}}+\bar{\pi}^{*}+\eta$

Observando-se a condição de equilíbrio intertemporal expressa em (3), constata-se a tendência à igualação de taxas reais de juros, acrescentando-se o prêmio de risco (uma variante da clássica "equação de Fisher") ${ }^{4}$ :

(8b) $r=\overline{r^{*}}+\bar{k}$

A equação (9) representa o equilíbrio no mercado cambial, com variação nula de reservas. Por seu turno, a equação (9a) consiste em sua aproximação log-linear. A variável $\overline{\mathrm{x}}$ é o log do saldo comercial autônomo (suposto positivo), $c_{1}$ é a elasticidade-renda das importações, $\mathrm{c}_{3}$ corresponde à elasticidade-renda das exportações, $\mathrm{c}_{2}$ consiste na elasticidade-câmbio do saldo comercial e, finalmente, $\mathrm{V}$ é a contrapartida do saldo comercial, com sinal inverso a este, na conta de capitais. Suponhamos $\mathrm{V}>0$, ou seja, a economia é importadora de capitais. $\mathrm{v}$ é o logaritmo de $\mathrm{V}$.

(9) $X\left(Y, S, \bar{Y}^{*}\right)+V=0$

(9a) $\bar{x}-c_{1} \cdot y+c_{2} \cdot s+c_{3} \cdot \bar{y}^{*}+v=0$

Dadás as suposições de baixa mobilidade de capital - com ausência de controle de capitais - e de constância do prêmio de risco, as necessidades de financiamento doméstico - renovação líquida de estoques de dívidas e novas vendas de títulos - dispõem da demanda ilimitada por parte de compradores de títulos, no país ou no exterior, à taxa de juros determinada em (8b). Basta supor algum mínimo custo de transação cambial para que a venda líquida de títulos ao exterior se acomode exatamente aos requisitos do saldo comercial, conforme (9). De qualquer forma, o equilíbrio no mercado de títulos financeiros é nossa equação redundante, já que a prevalência das demais condições de equilíbrio garantirá a igualdade entre compras e vendas de títulos financeiros.

As equações ou identidades (1b), (2a), (3), (4a), (5a), (6), (7a), (8b) e (9a) configuram um sistema de nove equações independentes, com 11 variáveis endógenas:

- variáveis reais: $\mathrm{y} ; \mathrm{r} ; \mathrm{s} ; \mathrm{v}$

- variáveis nominais (em nível): e; $\mathrm{p} ; \omega ; \mathrm{m} ; \mathrm{R}$

- taxas de variação: $\pi ; \eta$

${ }^{4}$ Voltaremos com mais detalhes deste ponto do modelo na seção 4.2. 
As variáveis reais podem ser determinadas de antemão. Independentemente do resto do sistema, a equação (8b) determina $r_{0}$, enquanto (4a) define $\mathrm{y}_{0}$. Com estas, a equação (1b) indica o $\mathrm{q}_{0}$. Finalmente, $\mathrm{v}_{0}$ pode ser obtido em (9).

Para a obtenção do restante, necessita-se fixar valores para duas entre as variáveis nominais e as taxas de variação. Chama-se de âncora nominal e de regime monetário-cambial justamente a essa escolha exógena de variáveis nominais a fixar, bem como de regras de procedimento quanto ao ajuste das variáveis monetárias e cambiais.

Em princípio, o governo pode tentar fixar qualquer uma das variáveis nominais. A âncora salarial, por exemplo, foi freqüentemente utilizada no Brasil dos anos 70 e 80, quando havia maior capacidade direta de influência do setor público na determinação de salários nominais básicos. Os regimes de política monetária que abandonaram simples metas monetárias em favor da administração de metas para as taxas de juros nominais constituem outro exemplo. O regime de metas de inflação focaliza a variável $\pi$.

No que segue, completamos o modelo com duas formas estilizadas de definir as âncoras cambial e monetária, a saber:

- âncora cambial:

(10a) $s=\bar{s}_{0}$

(11a) $\eta=(\mathrm{ds} / \mathrm{dt})=$ alguma constante $\bar{\lambda}_{\mathrm{s}}$

- âncora monetária:

(10b) $\mathrm{m}=\overline{\mathrm{m}}_{\mathrm{o}}$

(11b) $\mathrm{p}=\overline{\mathrm{p}}_{0} \mathrm{ou}(\mathrm{dm} / \mathrm{dt})=$ alguma constante $\bar{\lambda}_{\mathrm{m}}$

\section{2 Âncora cambial}

A âncora cambial envolve a determinação e a manutenção de um certo patamar para a taxa nominal de câmbio ou de sua evolução ao longo do tempo ou, ainda, faixas de variação (bandas cambiais) para aquela taxa. As bandas podem ser reajustadas periodicamente, mas o fato é que a flutuação da taxa de câmbio no mercado torna-se constrangida pelas metas, o que implica comprometimento do governo ou do banco central com a cobertura de excessos de demanda ou 
de oferta de moeda estrangeira, mediante uso de suas reservas. As equaçōes (10a) e (11a) completam o modelo, expressando uma âncora cambial e uma regra explícita adotada pela política cambial. Para simplificar suponhamos que $\bar{\lambda}_{\mathrm{s}}=0$.

A partir de (3), obtém-se $\pi_{0}=\bar{\pi}^{*}$, ou seja, no limite, a taxa de inflação doméstica se iguala à taxa de inflação no exterior (ou a $\bar{\pi}^{*}+$ $\bar{\lambda}_{\mathrm{s}}$ se esta última difere de zero). Da mesma forma, conhecendo-se $\mathrm{q}_{0}$, $s_{0}$ e $\bar{p}^{*}$, pode-se extrair $p_{0}$ de $(2 a)$. Note-se que, partindo inicialmente de uma situação de elevado diferencial positivo entre a inflação local e a do resto-do-mundo, caso a âncora cambial faça a inflação cair ao patamar de equilíbrio antes que a taxa real de câmbio elimine alguma valorização cambial real, o ajustamento do sistema exigirá deflação absoluta em p.

Os salários nominais também são obrigados a adaptar-se segundo a equação (5a). Da mesma forma adaptativa, o volume líquido de ingresso de capitais externos pode ser inferido do equilíbrio no mercado cambial (equação 9a). Com inflação e taxa real de juros definidas, a identidade (6) define a taxa nominal de juros.

Finalmente, constata-se que os volumes real e nominal dos meios de pagamento se tornam completamente endógenos, a partir da equação (7a), dados os níveis definidos para $\mathrm{p}_{0}, \mathrm{y}_{0}$ e $\mathrm{R}_{0}{ }^{5}$. A ancoragem cambial e a correspondente regra de política cambial acrescentam mais uma equação, uma variável endógena adicional (dm/dt) e uma regra monetária em nosso modelo:

(12a) $\mathrm{dm} / \mathrm{dt}=\pi_{0}=\bar{\pi}^{*}$

A ancoragem cambial implica abdicar de políticas monetárias ativas. Este é um resultado clássico dos modelos Mundell-Fleming com taxas fixas de câmbio. Sua viabilidade depende, é claro, de dois fatores:

- a retenção de estoques de moeda estrangeira mantidos como reservas do banco central vis-à-vis os picos na flutuação de demanda do mercado cambial; e

\footnotetext{
${ }^{5}$ Vale constatar que cada uma das equaçōes serviu para a determinação de alguma variâvel, não restando nenhuma equação redundante. Os passos seguintes seriam, evidentemente, examinar os limites de valores para os parâmetros que garantem o sentido econômico dos resultados do modelo, bem como examinar as condições de estabilidade no processo de ajustamento ao equilibrio. Para uma abordagem da dinâmica de ajustamento aos steady states com as âncoras monetária e cambial, em um modelo Mundell-Fleming com preços flexíveis, ver Maka (1997).
} 
- a capacidade de esterilização dos impactos monetários dos picos na oferta líquida positiva de divisas.

O que tende a acontecer nesta economia caso a adoção da âncora cambial se defronte com alguma inércia inflacionária, ou seja, com algum retardo no ajuste para baixo da taxa de inflação? Antes de tudo, valoriza-se em termos reais a taxa de câmbio (equaçōes 3 e 2a), com os correspondentes efeitos de redução no saldo comercial e de aceleração no endividamento externo (equação 9a). O equilíbrio no mercado de bens e serviços, por seu turno (equação 1b), passa a exigir queda na taxa real de juros ou a transformação da equação (4a), abrindo-se a possibilidade de $y \leq \bar{n}+\overline{I_{0}}$, ou seja, de desemprego.

A possibilidade da diminuição da taxa real de juros pode ser vista na equação (8a), a qual reflete os resultados da arbitragem entre taxas de juros nominais, independentemente de prevalecer ou não a condição efetiva de equilíbrio (8b). Com desvalorização zero, bem como taxa de juros externa e prêmio de risco constantes, pode acomodar-se a taxa local de juros reais. Neste caso, as necessidades de ingresso bruto de capital e endividamento externo bruto - ou de queima de reservas do banco central - passam a ter de cobrir não apenas o decréscimo no saldo comercial, como também, na presença de expectativas pessimistas quanto à sustentabilidade da política cambial, a fuga de capitais.

A taxa de juros nominal também desceria (equação 6), e o volume real de meios de pagamento teria de crescer acima da inflação resistente (equação 7a).

Embora a simplificação do modelo não permita examinar o papel dos salários no processo, pode-se presumir uma elevação no poder de compra dos assalariados, caso os reajustes nominais de salários continuem seguindo a inflação doméstica (conforme depreende-se de 5a), tendo em vista que ocorrerá redução nos preços relativos dos produtos importados presentes em sua cesta de consumo. Com isto, intensificam-se as pressões de queda no saldo comercial.

Enfim, o grau da defasagem cambial durante a adoção da âncora, com preços ou salários rígidos para baixo, poderá comprometer o próprio processo de ajuste:

(1) diante do desequilíbrio inicial, a transição para o nível real de equilíbrio na taxa de câmbio tende a exigir fortes reajustes dos salários nominais para baixo e/ou redução nas margens de lucro $(\bar{\tau})$;

(2) o aumento no endividamento externo líquido è a diminuição no saldo comercial, provocando além de tudo uma provável discre- 
pância crescente entre a desvalorização cambial efetiva e aquela tomada como inevitável pelo mercado, findam por elevar o prêmio de risco do país ( $\overline{\mathrm{k}}$ ). Neste caso, em seus estágios posteriores, a ancoragem cambial passa a subsistir apenas com taxas reais de juros mais altas que aquela do patamar inicial; e

(3) a despeito de, no início, maior consumo assalariado, queda na taxa real de juros doméstica e exercício de maior poder de compra em divisas (com a "absorção" doméstica subindo em relação à renda Gonçalves et al., 1998, p. 216-18), pode-se ter baixos investimentos durante o correspondente boom na economia, face à perda de competitividade da produção local e/ou à compressão de margens de lucro, bem como à ausência de credibilidade na sustentação da âncora. Da mesma forma, a subida nas taxas reais de juros nos momentos posteriores pode suscitar processos cumulativos de endividamento local - público e/ou privado - e subseqüentes elevações no prêmio de risco do país. Programas de estabilização cambial que suponham processos de ajustamento muito acentuado podem ver-se frustrados por seus próprios efeitos de "histerese" ${ }^{6}$ neste ajuste?

\footnotetext{
6 "Designamos por histerese o seguinte fenômeno: se uma economia passa de um estado E1 para um estado E2 quando uma variável v muda do valor v1 para o valor v2, ela não retorna a E1 quando v volta de v2 para v1, por causa de alteraçōes sofridas pelo sistema durante a situação E2" (Guillochon, 1993, p. 210). Dito de outro modo, após um choque, o estado final de repouso dependerá do que ocorre durante a trajetória de ajuste.

${ }^{7} \mathrm{~A}$ âncora monetária corresponde a fechar o modelo com a equação (10b) e uma das versões de (1 1b), liberando para flutuação a taxa nominal de câmbio. Caso a meta monetária seja estabelecida em termos reais, ou seja, em niveis de $(\mathrm{M} / \mathrm{P})_{0}$, a taxa de inflação é determinada a partir da taxa de juros nominal compatível com o equilíbrio no mercado monetário (ver equações 6 e 7a). Se, por

outro lado, a regra monetária assumida é (dm/dt) a uma taxa fixa $\bar{\lambda}_{\mathrm{m}}$, com $\mathrm{m}=\mathrm{m}_{\mathrm{o}}$ como ponto de partida, são ambos o nível de preços $\mathrm{p}_{0}$ e sua variação no tempo que se tornam endógenos $\left(\pi_{0}=\bar{\lambda}_{m}\right)$. Os resultados são idênticos com as duas formas de definição da âncora monetária. Diferentemente do caso da âncora cambial, no tocante à taxa nominal de câmbio tende a simplesmente prevalecer, em equilíbrio, a variação nominal do câmbio conforme os diferenciais de taxas de inflação, ou seja, a condição de equilíbrio (3): $\eta=\pi_{0}-\bar{\pi}^{\star}$. Constata-se como as demais variáveis nominais também são determinadas de modo endógeno no modelo: $\mathrm{s}_{0}$ por $2 \mathrm{a} ; \omega$ por $5 \mathrm{a} ; \mathrm{v}$ por $9 \mathrm{a}$. Vejamos se também podem ocorrer efeitos de histerese em programas de estabilizaçāo com base em âncoras monetárias, na presença de forte inércia inflacionária. Neste caso, o processo de ajustamento para baixo na inflação é buscado através de reduções significativas em $\bar{\lambda}_{\mathrm{m}}$.
} 
4 Inflação, produto e taxa de câmbio: uma aproximação de modelos de metas inflacionárias

\subsection{Uma primeira aproximação}

A implementação de um regime de metas inflacionárias supõe regras claras para definição das próprias metas, conforme um modelo de política monetária antiinflacionário ótimo. Neste caso, um ponto de partida é a curva de Phillips que, em uma forma log-linear, assume a seguinte expressão:

(13) $h_{t}=b\left(\pi_{t}-\pi_{t}^{e}\right)+u_{1}$

sendo que $h_{\mathrm{t}}$ é o hiato do produto, $\pi_{\mathrm{t}}$ é a taxa de inflação no período $t$, $\pi_{t}^{e}$ é a taxa de inflação esperada, e $u_{\mathrm{t}}$ representa o choque de oferta e o coeficiente b indica a elasticidade-preço da oferta agregada. Neste caso, as expectativas de inflação $\pi_{t}^{e}$ poderiam seguir um padrão adaptativo tal que:

$$
\text { (14) } \pi_{t}^{e}=(1-a) \pi_{t-1}+a \pi_{t-1}^{e}-v_{t}
$$

onde $0 \leq \mathbf{a} \leq 1 \mathrm{e} \mathrm{v}$ indica um choque aleatório.

Uma interessante abordagem de políticas monetárias sob condições de compromissos (commitments) e em caso de política ótima em condições discricionárias pode ser encontrado em Masson, Savastano $\mathcal{E}$ Sharma (1997). Neste caso, a equação de inflação poderia assumir duas formas gerais:

$$
\begin{aligned}
& \text { (15a) } \pi_{t}=\pi_{s t}^{e}+\frac{\lambda}{1+\lambda} \varepsilon \\
& \text { (15b) } \pi_{t}=\pi_{s t}^{e}+\lambda y_{t}^{e}+\frac{\lambda}{1+\lambda} \varepsilon
\end{aligned}
$$

onde $\pi_{\mathrm{st}}^{\mathrm{e}}$ é a taxa de inflação socialmente preferida, tal como uma expectativa de inflação, para um parâmetro $\lambda$, que representa a importância social relativa dada ao produto, ou ainda, o grau de aversão da sociedade à recessão temporária provocada por políticas antiinflacionárias (Simonsem, 1985). Note-se a que a diferença da equa-

\footnotetext{
${ }^{8}$ Para uma excelente revisão da dinâmica da inflação com expectativas adaptativas, com a descriçāo de um modelo de políticas antiinflacionárias ótimas, ver Simonsem (1985), cap. VIII.
} 
Ção (15a) para a equação (15b) é que a segunda sustenta uma maior aversão da sociedade à recessão temporária, tal como se expressa a constante $\lambda \mathrm{y}^{\mathrm{e}}$. Ou ainda, "This term in the policy rule is called the inflation bias because it adds a constant rate of inflation to the policy rule discretion - as proposed to the commitment case". (Green, 1996, p. 787).

Há, ainda, uma outra especificação para a Curva de Phillips com o propósito de estimar a taxa esperada de inflação, contudo, relacionando diretamente inflação atual e prevista com hiato de produto, com mudanças na taxa de câmbio real (Bogdanski et al., 2000). Deste modo, temos:

$$
\begin{aligned}
& \text { (16a) } \pi_{t}=\alpha_{1}^{b} \pi_{t-1}+\alpha_{2}^{b} \pi_{t-2}+\alpha_{3}^{b} h_{t-1}+\alpha_{4}^{b} \Delta\left(p_{t}^{F}+e_{t}\right)+\varepsilon_{t}^{b} \\
& \text { (16b) } \pi_{t}=\alpha_{1}^{f} \pi_{t-1}+\alpha_{2}^{f} E_{t}\left(\pi_{t+1}\right)+\alpha_{3}^{f} h_{t-1}+\alpha_{4}^{f} \Delta\left(p_{t}^{F}+e\right)+\varepsilon_{t}^{f} \\
& \text { (16c) } \pi_{t}=\frac{\left(\alpha_{1}^{f}+\alpha_{1}^{b}\right)}{2} \pi_{t-1}+\frac{\alpha_{2}^{f}}{2} E_{t}\left(\pi_{t+1}\right)+\frac{\alpha_{2}^{b}}{2} \pi_{t-2}+\frac{\left(\alpha_{3}^{f}+\alpha_{3}^{b}\right)}{2} \Delta\left(p_{t}^{F}+e_{t}\right)+\varepsilon_{t}
\end{aligned}
$$

sendo que a equação (16a) representa uma especificação do tipo Backward-looking, motivada pela suposição de expectativa adaptativa, a equação (16b) representa uma especificação do tipo Forward-looking, com o objetivo de captar o parâmetro de instabilidade presente em quebras estruturais, e a equação (16c) expressa a combinação entre estas duas especificações, onde se encontra um equilíbrio adequado entre componentes de persistência da inflação com componentes expectacionais. Há, ainda, a preocupação quanto à transferência de mudanças na taxa de câmbio sobre inflação doméstica (passthrough ratio), se se constitui em uma constante a partir da estimação de uma média do comportamento passado, ou se equivale a uma transferência quadrática qualquer com suas variantes.

Seguindo o campo do exercício empírico, há um conjunto significativo de metodologia de tratamento do regime de metas de inflaçăo, como os trabalhos de Brunila $\mathcal{E}$ Lahdenperä (1995), Bernanke (1999), Haldane $\mathcal{G}$ Salmon (1995), Yales (1995), Holffmaister(1999) e Christoffersen $\mathcal{E}$ Wescott (1999). Mesmo com a preocupação voltada seja para o processo de implementação da estratégia de metas de inflação, seja para análise de sua eficácia absoluta ou em comparação com outras estratégias de metas, via de regra, as especificaçōes das metodologias de tratamento empírico estão associadas a algum processo de ajustamento às informações passadas. Noutras palavras, estima-se, em geral, um modelo IS-LM backward-looking com uma curva 
de Phillips com expectativas adaptativas. Em alguns casos, há uma nítida preocupação com alguma regra de feedback da política monetária seguindo um processo estocástico em que a taxa de juros ( $\left.\Delta \Delta_{t}\right)$ se ajusta com o desvio da inflação em período passado (observada) com a meta de inflação $\left(\pi^{\text {targ }}\right)$, assim expressa:

$$
\begin{aligned}
& \Delta \mathrm{i}_{\mathrm{t}}=\lambda\left(\Delta \mathrm{p}_{\mathrm{t}-1}-\pi^{\mathrm{targ}}\right) \\
& \Delta \mathrm{i}_{\mathrm{t}}=\lambda\left(\mathrm{E}_{\mathrm{t}-1}^{\mathrm{e}}\left[\Delta p_{\mathrm{t}-1} \mid \Omega_{\mathrm{t}}\right]-\pi^{\mathrm{targ}}\right)
\end{aligned}
$$

onde E é o operador esperança e o sobrescrito $e$ expressa a opinião do Banco Central comparada com a opinião do setor privado da economia.

\subsection{Taxa de câmbio, taxa de juros e metas de inflação}

O problema passa a se voltar para o comportamento da taxa de câmbio e da taxa de juros, sob metas de inflação. Assim, a pergunta é: Qual deve ser a regra do Banco Central para atuar sobre o mercado de câmbio e sobre a formação da taxa de juros, em um regime de taxa de câmbio flexível, possivelmente administrada, e com meta inflacionária? Uma especificação mais simples para a taxa de câmbio poderia associar-se ao modelo de paridade de taxa de juros, conforme algum mecanismo do tipo Efeito-Fisher'. Assim,

(18) $\left(s_{t}^{e}-s_{t}\right) / s_{t}=\left[\left(e_{t}^{e}-e_{t}\right) / e_{t}-\left(\pi_{t}^{e}-\pi_{t}^{e^{*}}\right)\right.$

sendo que o lado esquerdo da equação expressa a taxa de variação real da taxa de câmbio, enquanto que o primeiro componente do lado direito da equação expressa a taxa de mudança da taxa de câmbio nominal, e o segundo componente mostra o diferencial entre a inflação doméstica esperada e a inflação externa esperada.

Uma vez que, do ponto de vista do investidor estrangeiro, o diferencial entre a taxa de juros real doméstica e a taxa de juros nominal externa $\left(i_{t}-i_{t}^{*}\right)$ é igual à taxa de variação da taxa de câmbio nominal:

(19a) $i_{t}-i_{t}^{*}=\left(e_{t}^{e}-e_{t}\right) / e_{t}$

Substituindo (18) em (19a), obteremos:

9 Segundo o Efeito Fisher, qualquer aumento na taxa de inflação esperada que não altere a taxa de juros real esperada deve se refletir na taxa de juros nominal. 
(19b) $i_{t}-i_{t}^{*}=\left[\left(s_{t}^{e}-s_{t}\right) / s_{t}+\left(\pi_{t}^{e}-\pi_{t}^{e}\right)\right.$

Como em regime de metas de inflação é possivel supor que a paridade de poder de compra pode operar adequadamente, $s_{t}^{e}=s_{t}$, tal que (19b) se reduz à seguinte expressão:

(19c) $i_{t}-i_{t}^{*}=\left(\pi_{t}^{e}-\pi_{t}^{e}\right)$

Ou seja, a diferença entre as taxas de juros doméstica e estrangeira é igual à diferença entre as taxas de inflação nacional e externa esperadas. Como a taxa de juros real esperada $r_{t}^{e}$ é igual à taxa de juros nominal i menos a taxa de inflação esperada, $\pi_{t}^{e}$ :

(20) $r_{t}^{e}=i_{t}-\pi_{t}^{e}$

de modo que:

(21a) $\left(r_{t}^{e}-r_{t}^{e^{*}}\right)=\left(i_{t}-\pi_{t}^{e}\right)-\left(i_{t}^{*}-\pi_{t}^{e^{*}}\right)$

ou ainda,

(2Ib) $\left(r_{t}^{e}-r_{t}^{e^{*}}\right)=\left(s_{t}^{e}-s_{t}\right) / s_{t}$

A partir deste modelo, há dois complementos que devem ser levados em conta no caso do regime de metas de inflação. Primeiro, há que se definir a presença de algum prêmio de risco cambial, preocupação recorrente em modelos para economias emergentes; segundo, é preciso especificar o modelo para uma taxa de inflaçāo doméstica esperada igual à meta de inflação, ou seja, $E\left(\pi_{t} \mid \Omega_{t}\right)=\pi^{\text {targ }}$. No primeiro caso, a taxa de juros em moeda doméstica deve ser igual à taxa de juros em moeda estrangeira mais um prêmio futuro da moeda estrangeira em relação à moeda doméstica:

(22) $i_{t}=i_{t}^{*}+\left(f_{t}-e_{t}\right) / e_{t}$ onde $f_{t}$ é a taxa de câmbio futura, ou a taxa de câmbio acordada no período corrente para contratos com vencimento em período futuro.

Deste modo, observado o efeito-Fisher e a possibilidade de um prêmio de risco, o comportamento da taxa de câmbio, sob regime de metas de inflação, poderia se expressar como segue:

(23) $E\left(s_{t} \mid \Omega_{t}\right)=(1+\lambda)^{-1} \sum_{i=0}^{\infty}\left[\frac{\lambda}{1+\lambda}\right]^{j} E\left(W_{t+1}\right)$ 
para $\Omega_{\mathrm{t}}$ um conjunto de informações, $\lambda /(1+\lambda)$ um fator de desconto, e para

$$
\text { (24) } E\left(W_{t+1}\right)=\left(r_{t}^{e}-r_{t}^{e}\right)+\left(f_{t}-e_{t}\right) / e_{t}
$$

De uma perspectiva de longo prazo, observada uma possível trajetória da taxa de câmbio em direção a algum fundamento econômico, tem-se que seus desvios de curto prazo podem ser explicados pela seguinte expressão:

$$
\text { (25) } E\left(s_{t}-s_{t}^{L P} \mid \Omega_{t}\right)=(1+\lambda)^{-1} \sum_{i=0}^{\infty}\left[\frac{\lambda}{1+\lambda}\right]^{j}(1 / \omega) E\left(W_{t+1}\right)
$$

de modo que o desvio da taxa de câmbio de seu nível de equilíbrio de longo prazo é proporcional ao diferencial de taxa de juros real somado ao prêmio de risco cambial, e a velocidade de ajustamento à trajetória de longo prazo é definida por $(1 / \omega)$. Noutras palavras, quanto mais a taxa de juros real doméstica se distanciar da taxa de juros real externa, dado um prêmio de risco cambial, mais a taxa de câmbio real estará fora de seu nível fundamental. Fixada a meta de inflação, a taxa de juros nominal doméstica deverá se ajustar à taxa de juros nominal externa, dada a taxa de inflação esperada estrangeira e o prêmio de risco. Exceto em caso de convergência crível da taxa de inflação doméstica à taxa de inflação externa, em geral bem mais baixa do que a doméstica, o modelo de metas de inflação implica uma inexorável taxa de juros doméstica mais elevada do que a taxa de juros externa.

\section{Considerações Finais}

A estratégia de metas inflacionárias está fortemente associada à escolha de regimes de câmbio mais flexíveis, e se constitui em sucesso em estabilizar o comportamento de preços em níveis relativamente baixos, o que implica alguma trajetória de crescimento do produto, mas não necessariamente com taxa de juros baixas, e taxa de câmbio relativamente pouco volátil. Após a apresentação das situações de equilibrio nos diversos mercados (de bens e serviços, monetário, cambial e de títulos financeiros, de trabalho, passamos para a definição, de forma estilizada, das âncoras cambial e nominal, conforme configuração típica de um modelo Mundell-Fleming.

Em seguida, pudemos realizar uma primeira aproximação da dinâmica da inflação, em condiçōes de uma política monetária ótima, sob o regime de flutuação cambial com metas inflacionárias. Atesta- 
mos que, em geral, o modelo de metas de inflação é uma extensão do modelo Mundell-Fleming, com trajetórias para o comportamento do produto subordinadas à trajetória da inflação, com forte componente expectacional na definição da trajetória conjunta entre taxa de juros e taxa de câmbio, guardado um prêmio de risco.

Neste sentido, um modelo de flutuação cambial sob metas inflacionárias pode se apresentar como mais flexível e mais crível que modelos do tipo âncoras cambiais, mais bem coordenado pelas Autoridades Monetárias e com maior autonomia de política monetária que regimes de câmbio mais fixos, entre outros. Dificilmente se apresentam como modelos responsáveis pela maior variabilidade do produto, mesmo que não se constituam em condição absoluta para a sustentação de trajetórias estáveis para a inflação, ou que não impliquem queda da taxa real de juros. 


\section{Anexo: Estratégias de Políticas Monetárias (1999)}

\begin{tabular}{|c|c|c|c|c|c|}
\hline $\begin{array}{l}\text { Regimes } \\
\text { Cambiais } \\
\text { Número de }\end{array}$ & \multicolumn{5}{|c|}{ Marco de Política Monetária } \\
\hline & Âncora Cambial & $\begin{array}{l}\text { Objetivos } \\
\text { Agregados } \\
\text { Monetários }\end{array}$ & $\begin{array}{l}\text { Estratégia } \\
\text { Baseada } \\
\text { em Objetivos } \\
\text { de Inflação }\end{array}$ & $\begin{array}{l}\text { Programa } \\
\text { Respaldado } \\
\text { pelo FMl ou } \\
\text { outro } \\
\text { Programa }\end{array}$ & Outro \\
\hline $\begin{array}{l}\text { Taxa de } \\
\text { Câmbio dentro } \\
\text { de bandas } \\
\text { móveis } \\
(7) \\
\text { Exemplos }\end{array}$ & $\begin{array}{ll}\text { (4) } & (3) \\
\text { Honduras* } & \text { Uruguai* } \\
\text { Israel* }^{*} & \text { Venezuela }\end{array}$ & Siri Lanka* & $\begin{array}{l}\text { (2) } \\
\text { |srael* } \\
\text { Polônia* }\end{array}$ & Uruguai* $^{*}{ }^{*}$ & \\
\hline $\begin{array}{l}\text { Flutuação } \\
\text { administrada } \\
\text { sem uma de } \\
\text { taxa } \\
\text { de câmbio } \\
\text { previamente } \\
\text { anunciada } \\
(26) \\
\text { Exemplos }\end{array}$ & & $\begin{array}{l}\text { (3) } \\
\text { Eslovénia } \\
\text { Jamaica } \\
\text { RDP Lao }\end{array}$ & $l_{\text {Rep }}$ Checa & $\begin{array}{l}(10) \\
\text { Croácia } \\
\text { Paquistão } \\
\text { Etiopia }\end{array}$ & $\begin{array}{l}\text { (12) } \\
\text { Paraguai3 } \\
\text { Guatemala3 } \\
\text { Nigéria3 }\end{array}$ \\
\hline $\begin{array}{l}\text { Flutuação } \\
\text { independente } \\
\text { (5i) } \\
\text { Exemplos }\end{array}$ & & $\begin{array}{l}\text { (15) } \\
\text { Colômbia } \\
\text { Perü* } \\
\text { Coréig** } \\
\text { Guinét }^{*}\end{array}$ & $\begin{array}{l}\text { (9) } \\
\text { Brasil } \\
\text { Chile5 } \\
\text { Canadá } \\
\text { Nova } \\
\text { Zelândia } \\
\text { Reino Unido } \\
\text { Suécia } \\
\text { Austrólia } \\
\text { Kazajstão } \\
\text { Angola }\end{array}$ & $\begin{array}{l}\text { (27) } \\
\text { Brasil } \\
\text { Albânia } \\
\text { Armênia } \\
\text { Coréia* } \\
\text { Filipinas" } \\
\text { Indonésia } \\
\text { Madagascar } \\
\text { México } \\
\text { Peru } \\
\text { Rússia3 } \\
\text { Tailândia }\end{array}$ & $\begin{array}{l}\text { (10) } \\
\text { Estados } \\
\text { Unidos3 } \\
\text { Equador3 } \\
\text { Japão3 }\end{array}$ \\
\hline
\end{tabular}

** MCCO: Banco Central do Caribe Oriental; UEMOA: União Esconômica e Monetária da África Ocidental; CEMAC: Comunidade Econômica e Monetária da África Central

1) O asterisco indica que o país adota mais de uma âncora nominal na execução da política monetária.

2) Estes países também estabeleceram um regime de caixa de conversão (currency board) dentro do mercado comum.

3) As Autoridades Monetárias não adotam uma âncora nominal explícita, porém controlam vários indicadores na execução da política monetária.

4) Até meados de 2002, as moedas nacionais manterão seu status legal em seus territórios nacionais.

5) Há um acordo cambial entre os países membros.

6) O país estabeleceu de fato regime de câmbio flutuante administrado ou independente anunciado oficialmente. Na Jordânia, a moeda está vinculada oficialmente ao DES, porém, de fato, ao dólar dos EUA.

7) As taxas de câmbio são determinadas numa relação rígida com o DES, dentro de margens de até 7,25\%; entretanto, devido às relaçōes com o dólar EUA estas margens nem sempre são respeitadas. Fonte: IMF (1999). International Financial Statistics, dezembro de 1999 (Anuário). 


\section{Referências Bibliográficas}

BARRO, Robert e GORDON, David (1983). "A positive theory of monetary poly in a natural rate model". Journal of Political Economy, 91(4).

BERNANKE, Bem S. et al. (1999). Inflation Targeting: lessons from the Internantional Experience. Princeton, UP.

BLANCHARD, Olivier e FISCHER, Stanley (1989). Lectures on Macroeconomics. MIT Press.

BRUNILA, Anne e LAHDENPERÄ, Harri (1995). "Inflation targets: principal issues practical implementation". Bank of England (1995). Targeting Inflation, England, march 1995.

CHRISTOFFERSEN, Peter e WESCOTT, Robert (1999). "Is Poland ready for inflation targeting?". IMF Working Paper, WP/99/41, IMF: Washington, March 1999.

DEBELLE, Guy (1997). "Inflation Targeting in Practice". IMF Working Paper, WP/97/35, IMF: Washington, March 1997.

DORNBUSCH, Ruger (1976). "The theory of flexible exchange rate regime and macroeconomic policy". A. Chrystal (ed.). Monetarism, v. 2. Aldershot: Edward.

GONÇALVES, Reinaldo et al. (1998). A nova economia internacional: uma perspectiva brasileira. Rio de Janeiro: Campus, 1998.

GREEN, John (1996). "Inflation targeting: theory and policy implications". IMF Staff Papers, 43(4), december, 1996.

GUILLOCHON, B. (1993). Économie internationale. Paris: Dunod, 1993.

HALDANE, Andrew e SALMON, Christopher (1995). "Three issues on inflation targets". Bank of England (1995). Targeting Inflation, England, march 1995.

HOFFMAISTER, Alexander (1999). “Inflation targeting in Korea: an empirical exploration". IMF Working Paper, Washington, IMF, WP/99/7.

JADRESIC, Esteban (1999). "Inflation Targeting na Output Stability". IMF Working Paper, WP/99/61, IMF: Washington, April 1999.

KENEN, Peter (1998). Economia Internacional: teoria e política. Rio de Janeiro, Campus.

KYNDLAND, Finn e PRESCOTT, Edward (1977). "Rules rather than discretion: the inconsistency of optimal plans". Journal Political Economy, 85(3).

MASSON, Paul R., SAVASTANO, Miguel A. e SHARMA, Sunil, (1997). "The Scope for Inflation Targeting in Developing Countries". IMF Working Paper, WP/97/130, IMF: Washington, October 1997.

MAKA, A. (1997). "Estratégias de combate à inflação: âncora cambial versus âncora monetária". In: Anais XXV Encontro Nacional de Economia, Recife, 1997.

Recife: ANPEC, dez. 1997. v. 1, p. 61-81. 
MCCALLUM, B. T. (1996). International monetary economics. Oxford: Oxford Univ. Press, 1996.

MISHKIN, Frederic (2000). Monetary policy strategies for Latin America. NBER, Draft, February, 22, 2000.

NETTO, Antônio D. (1999). "Sobre as metas inflacionárias". Economia Aplicada, São Paulo: FEA-USP/FIPE, 3(3), julho-setembro de 1999.

SICSÚ, João (2000). "Teoria e evidências do regime de metas inflacionárias: algumas observações críticas preliminares”. Revista de Economia Política, São Paulo: Nobel (forthcoming).

SIMONSEN, Mário H. (1983). Dinâmica Macroeconômica. São Paulo: McGrawHill.

YALES, Anthony (1995). "On the design of inflation targets". Bank of England (1995). Targeting Inflation, England, march 1995. 